\title{
Evaluating Recognition Memory in Children Referred for Suspected Obstructive Sleep Apnea
}

\begin{abstract}
Keywords: Sleep disordered breathing; Children; Facial recognition memory; Neurobehavioral performance

\section{Abstract}

Background: A majority of previous studies showing memory deficits in children with Obstructive Sleep Apnea (OSA) have focused on recall memory tasks and have relied upon subjective measures of sleep (parental reported sleep or actigraphy). The present study uses polysomnography (PSG) to confirm OSA the night following the learning situation. Our objective was to assess the extent of memory deficits in children referred for suspected OSA.
\end{abstract}

Methods: Children with suspected OSA were recruited prior to a night of PSG. Children performed a brief facial recognition memory task: 1) Prior to bedtime in the sleep laboratory, the child was instructed to study faces; 2) In the morning, the child was presented with previously studied faces and foils not seen before. The child was asked to identify which faces they remembered. We assessed memory variables accuracy (\%correct) and reaction time (RT, milliseconds) and sleep/respiratory metrics.

Results: Twenty-two children (13.1 years+/-2.9) were included. Those with OSA (mixed/obstructive apnea-hypopnea index, $M O A H I \geq 1)$ vs. without OSA (MOAHI<1) had more respiratory-related arousals $(p=0.02)$ but no difference in the oxygen desaturation indices. Regardless of OSA status, children showed greater accuracy in recognizing the faces studied compared to the foils $(p=0.001)$. Pearson correlations revealed no associations between memory performance and respiratory variables (MOAHI, respiratory arousals, desaturations). There were no associations between RT and sleep variables (sleep staging and total sleep time). There was an association between increased sleep and better accuracy in memory performance $(r=0.45$, $p=0.04)$

Conclusion: Our data suggest that OSA in children may not be associated with deficits in memory performance, when using a brief recognition memory task. We found a modest association between increased sleep duration and higher accuracy in memory performance. Future studies should determine whether sleep quantity in OSA is relevant in preventing a child's susceptibility to impairments in recognition and other types of memory.

\section{Introduction}

Sleep-disordered breathing (SDB) is a common disorder during childhood, with a spectrum of severity ranging from snoring to severe obstructive sleep apnea (OSA) [1,2]. In children with SDB, oxygen levels in the blood can drop (hypoxia), often disrupting sleep [35] and contributing to various negative outcomes such as daytime sleepiness, hyperactivity, and problems with memory. The mechanism that links SDB to deficits in memory is not completely understood. One theory proposes that the combination of sleep disruption and hypoxia related to SDB may produce behavioral deficits and memory problems [6]. This theory is further supported by the finding that treatment of SDB often improves deficits in memory and reverses behavioral symptoms [7-9].

Much of the information regarding the neurocognitive
Journal of

Neurology and Psychology

\section{Melodee A. Mograss ${ }^{1,2,3 *}$, Elise Mok $^{4}$ and Evelyn Constantin $^{4,5 *}$}

${ }^{1}$ Department of Psychology, Concordia University, Montreal, QC, Canada

${ }^{2}$ Department of Exercise Science \& Psychology, Concordia University, Montreal, QC, Canada

${ }^{3}$ Concordia University PERFORM Centre, Montreal, QC, Canada

${ }^{4}$ The Research Institute, McGill University Health Centre (RIMUHC), Montreal, QC, Canada

${ }^{5}$ Department of Pediatrics, Montreal Children's Hospital/McGill University Health Centre, Montreal, QC, Canada

\section{*Address for Correspondence}

Melodee A. Mograss, Ph.D, Department of Exercise Science and Psychology, Concordia University, Montreal, QC, Canada, E-mail: melodee.mograss@concordia.ca

Evelyn Constantin, MD MSc(Epi), Montreal Children's Hospital, Pediatric Sleep Laboratory, 2300 rue Tupper, Room C-508, Montreal (Quebec) H3H 1P3, Tel: (514) 412-4321; Fax: (514) 412-4234; E-mail: evelyn.constantin@mcgill.ca

Submission: 21 July 2015

Accepted: 11 August 2015

Published: 15 August 2015

Copyright: (๑) 2015 Mograss MA, et al. This is an open access article distributed under the Creative Commons Attribution License, which permits unrestricted use, distribution, and reproduction in any medium, provided the original work is properly cited.

Reviewed \& Approved by: Dr. Sefa Bulut, Associate Professor, Department of Educational Sciences and Head of Counseling Psychology and Guidance Program, College of Education, Abant Izzet Baysal University, Turkey

consequences of SDB in children relates to disease at the severe end of the clinical spectrum [10,11]. In more severe forms of SDB, particularly OSA, long-term recall on a pictorial task have been reported to be worse compared with controls [12]. In children with milder forms of OSA, sleep and memory studies reveal lower scores on neurocognitive tests of short-term memory $[13,14]$, with significant improvement in scores after adenotonsillectomy $[7,15]$. Other researchers have either failed to replicate the positive findings or report conflicting results $[9,16,17]$.

A majority of the previous studies focus on recall and relied upon parental reported sleep variables or actigraphy measures. In the few studies that used PSG, often the sleep period occurred weeks after the task was administered instead of following the learning situation. The present study addresses these limitations. Our rationale for the study is that little is known about the risks of "recognition" memory (i.e. recognizing previously encountered items) deficits in children with OSA and how memory deficits vary in relation to OSA status. The current study evaluates recognition memory and uses an objective sleep measure (PSG) right after the learning situation. Thus, the goal of this study is to address the association between recognition memory deficits and OSA status in children referred for suspected OSA. 
Citation: Mograss MA, Mok E, Constantin E. Evaluating Recognition Memory in Children Referred for Suspected Obstructive Sleep Apnea. J Neurol Psychol. 2015;3(2): 6.

ISSN: 2332-3469

\section{Methods}

\section{Participants}

We prospectively recruited children aged 7-17 years from the pediatric sleep laboratory at our institution who were referred for suspected OSA. Parent(s)/guardian were asked to complete a sleep questionnaire on their child's medical history and sleep history [18]. Children were excluded if they had a past or current history of psychiatric conditions, known sleep disorder, craniofacial, genetic, or neurologic disorder or if they had undergone a prior adenotonsillectomy. Each potential subject had previously undergone an overnight home oximetry screening. The oximetry test was interpreted as inconclusive for obstructive sleep apnea and thus these children were scheduled for a PSG, the definitive gold standard diagnostic tool for obstructive sleep apnea. The study was approved by the Institutional review board at the Montreal Children's Hospital/ McGill University Health Centre.

\section{Demographic information, sleep history}

We collected data from demographic and sleep questionnaires completed by the parent(s)/guardian before the initial home oximetry screening. These questionnaires were collected for screening and descriptive purposes.

\section{Sleepiness \& vigilance scales}

We assessed daytime sleepiness and alertness using the Epworth Sleepiness Scale (ESS) and a Visual Analog Scale (VAS). The ESS measures daytime sleepiness or sleep propensity with versions validated in English [19] and French [20]. The ESS was modified to be more applicable to children. The mention of alcohol was excluded in question number 7 , and question 8 was taken to indicate that if the youth did not drive s/he was a passenger in the car. ESS total scores ranged from 0 to 24 . A score of $>10$ is considered consistent with excessive daytime sleepiness.

Self-ratings of alertness (Visual Analog Scale, VAS) were measured twice: in the evening prior to the child studying the faces (stimuli) and then again in the morning before taking the memory task. The VAS for alertness consists of a $100 \mathrm{~mm}$ line with each end of the line corresponding to "very sleepy" to "very alert". The child indicates a position on the $100 \mathrm{~mm}$ line that best describes how they feel at that moment prior to taking the memory task. Results are expressed in distance (in $\mathrm{cm}$ ) between left end of the scale and the bar drawn by the participant.

\section{Facial recognition memory task}

We used a facial recognition memory task [21-23] whereby the stimuli (photographs) were presented on a computer screen (E-Prime v2.0 software). These face stimuli were taken from the MED face bank, which is composed of front-view color photographs of faces taken under the same conditions (background and light) [24]. The entire set of stimuli included 120 faces, 80 faces were presented at both the study session (prior to bedtime) and test session (the morning after sleep, after PSG); 40 other faces were used as foils (new) during the test session.

The children performed a brief practice session before the actual memory task. Prior to bedtime, the child was shown a series of face photographs and instructed to study them for a subsequent test. In the morning, the child's task was to indicate as accurately and quickly as possible the previously presented faces by pressing a key ( $1=$ yes, $2=$ no) on the computer keyboard. Each session lasted about 15 minutes and was separated by an 8-hour delay of a night's sleep in the laboratory. The memory task variables included reaction times, RT (msec) and accuracy (\% correct).

\section{Polysomngraphy, PSG}

We performed an overnight PSG in the laboratory on children with inconclusive oximetry studies to confirm OSA. The PSG recording included respiratory metrics using respiratory inductive plethysmography and nasal pressure, oxygenation with pulse oximetry (Masimo ${ }^{\circledR}$ Radical-7, software version 7801) and sensor (LNC neo-3 adhesive sensors), electromyelography (chinEMG), electrocardiogram, and electroencephalography (EEG), electrooculography for sleep/wake determination. In addition, audiovisual recordings were made to monitor snoring, movements, and arousals and to confirm sleep staging [25].

Indices of apneas and hypopneas (AHI - number of all apneic and hypopneic events per hour of sleep and MOAHI - number of mixed/obstructive apneic and hypopneic events per hour of sleep) were determined with PSG. Using standard pediatric criteria [26], we defined OSA as a MOAHI $\geq 1$ events per hour) on overnight PSG. Sleep architecture was scored in $30 \mathrm{~s}$ epochs according to the criteria outlined by Rechtschaffen and Kales [27]. Sleep duration was limited by having the parent(s) and child arrive in the laboratory at $\sim 19 \mathrm{~h} 30$ (bedtime $\sim 21$ h00) with a scheduled wake time at between 5:00-5:30 a.m., which was similar to the child's sleep diary weekday wake time.

\section{Data analysis}

Continuous data are presented as means with standard deviations (SD). Categorical data from the pre-screening questionnaire, demographics and behavioral symptoms are reported as frequencies and percentages. For analysis purposes, the children were separated into two groups based on the PSG: OSA defined as a MOAHI $\geq 1 / \mathrm{h}$ and No OSA, a MOAHI $<1 / \mathrm{h}$.

The scale for alertness (VAS) administered prior to each session and the pre-screening sleepiness scale (ESS) was used for descriptive purposes and to compare the groups (OSA, No OSA) at baseline.

Student $\mathrm{t}$-tests were performed to compare the two groups on the respiratory variables [MOAHI, AHI, respiratory arousals index, oxygen desaturation index ( $\mathrm{SpO} 2$ index), minimum $\mathrm{SpO} 2(\%)]$. Other sleep variables were Total Sleep Time, TST (h) - amount of sleep during the PSG recording; Stage 1(\%); Stage 2 (\%); slowwave sleep, SWS (\%) - combined Stage 3 \& Stage 4; rapid eyemovement sleep, REM (\%); Sleep Onset - lights out to any sleep stage; Awakenings (number); Sleep Efficiency (\%); wake after sleep onset, WASO - minutes of wake ( $>15 \mathrm{~min}$ ) occurring during sleep onset to sleep offset.

Performance on the memory task [reaction times, RTs (msec) and accuracy (\%correct)] obtained in both groups for studied stimuli (referred to as old) and the foils (new) were compared by mixed ANOVA on groups (OSA, No OSA) by condition ("old" stimuli, "new" stimuli). RTs were reported on the correct responses. 
Citation: Mograss MA, Mok E, Constantin E. Evaluating Recognition Memory in Children Referred for Suspected Obstructive Sleep Apnea. J Neurol Psychol. 2015;3(2): 6.

ISSN: 2332-3469

Pearson Product-Moment correlations were used to determine if there was a relationship between the following variables: 1) memory performance (RT, Accuracy) and MOAHI, 2) memory performance and respiratory variables (see above) and 3) memory performance and sleep variables (see above) assessed from PSG. Two-tailed tests were used. A p-value of less $<0.05$ was considered significant. Statistical analyses were conducted with SPSS (version 16 Chicago, USA).

\section{Results}

\section{Subject characteristics}

We collected data on a total of 23 children referred to the pediatric sleep laboratory for evaluation of OSA. One child was excluded due to an illness on the night of the PSG. Subjects were classified in their respective groups (OSA: MOAHI $\geq 1 / \mathrm{h}$; No OSA: MOAHI $<1 / \mathrm{h}$ ) based on the PSG.

A total of 22 children aged 13.1+/-2.9 yrs participated in the study (Table 1). Although the children with OSA compared to those without were 2.1 yrs older, there was no statistically significant difference in age. The children with a MOAHI $\geq 1 / h(n=5)$ compared to those with a MOAHI $<1 / \mathrm{h}(\mathrm{n}=17)$ had slightly higher proportions of males (Table 1).

Of the 22 children studied, $73 \%$ were overweight with a body mass index (BMI) of $>97 \%$ ile. Parents indicated that their child had symptoms consisting of daytime sleepiness (5\%), poor attention (14\%) restless sleep/awakenings (18\%) or hyperactivity/aggressive behaviors (14\%) in the range of most-to-all of the time. Forty percent of parents reported that their child had loud snoring and 38\% reported mouth breathing during sleep (most-to-all of the time).

\section{Polysomnography data}

The respiratory data and the sleep data from the overnight PSG studies for our study population are shown in Table 2. The MOAHI was significantly different for those with OSA compared to those without OSA ( $p=0.03)$. Similarly, the AHI was different for those with OSA compared to those without ( $\mathrm{p}=0.02)$, as was the number of respiratory-related arousals $(\mathrm{p}=0.02)$ between the two groups. There was no difference between the two groups on oxygen desaturation $\mathrm{SpO} 2$ index $(\mathrm{p}=0.06)$. The average minSpO2 levels for the OSA group were no different than those for children without OSA ( $\mathrm{p}=0.15)$.

There were no statistical differences between the two groups on the following sleep variables [TST; Stages 1(\%), Stage 2 (\%),
SWS (\%) and REM (\%); Sleep Efficiency (\%), Sleep Onset; number of Awakenings] assessed by PSG. However, there was a significant difference in the amount of wakefulness during sleep, WASO; $\mathrm{p}=0.01$ (see Table 2).

There was a difference in the children's ability to accurately recognize faces studied prior to bedtime (Old) and the "foils" (New). Regardless of the OSA status, the mean number of studied faces remembered were greater than those faces not studied $(\mathrm{N}=22$, Old: $\mathrm{M}=40.0 \mathrm{SD}=8.2$ vs. New stimuli: $\mathrm{M}=29.1 \mathrm{SD}=3.6$ ). However, we found no difference in accuracy between the groups of children (OSA versus No OSA) on recognition memory. As illustrated in Figure 1 bottom, there were significantly more accurate responses for Old vs. New $(F(1,20)=19.4, p=0.001)$, but no significant interaction was seen between Old/New stimuli and the group factor, $F(1,20)=0.12 ; p>0.05$ (Figure 1).

There were no significant differences found in reaction times, that is, how quickly the children responded to seeing those stimuli studied before sleep (Old) compared to the "foils" (New) [Old: $1700.6+/$ $599.3 \mathrm{~ms}$ vs. New RTs: $1758.2+/-602.2 \mathrm{~ms},(\mathrm{~F}(1,20)=0.92, \mathrm{p}>0.05)]$. Furthermore, we found no effect of group (OSA versus No OSA) on reaction times. In addition, there was no significant interaction involving RTs (Old, New) and the group factor $(\mathrm{F}(1,20)=0.79$, $\mathrm{p}>0.05$ (not shown).

Accuracy performance and PSG sleep variables: We found greater accuracy was associated with increases in the amount of sleep (TST) (Pearson: $\mathrm{r}=0.45, \mathrm{p}<0.04$ ), indicating that as sleep duration increased, memory performance improved in the children (see Figure 2). There were no significant correlations between improvements in accuracy and SWS (\%) ( $\mathrm{r}=0.14$, NS) or REM (\%) ( $\mathrm{r}=0.34$, NS) nor were there correlations between accuracy and Awakenings or WASO ( $\mathrm{p}>0.05$, not shown).

RTs performance and PSG sleep variables: There were no significant correlations between improvements in RTs on the correct responses and any of the following sleep variables (TST, SWS, REM, Awakenings, WASO).

Performance and severity of OSA parameters: No significant correlations were identified between performance (Accuracy, RTs) and indicators of respiratory severity as measured by MOAHI, respiratory arousals, $\mathrm{SpO} 2$ Index and sleep disruption (WASO and Awakenings).

Table 1: Parental report of child's characteristics on pre-screening questionnaire.

\begin{tabular}{|c|c|c|c|}
\hline & $\begin{array}{c}\text { Total } \\
\text { Subjects } \\
(\mathrm{N}=22)\end{array}$ & $\begin{array}{c}\text { OSA } \\
(\mathrm{N}=5)\end{array}$ & $\begin{array}{c}\text { No OSA } \\
(\mathrm{N}=17)\end{array}$ \\
\hline Age, y (mean $\pm S D)$ & $13.1 \pm 2.9$ & $14.7 \pm 1.8$ & $12.6 \pm 3.0$ \\
\hline Male gender $\mathrm{n}(\%)$ & $14(64 \%)$ & $4(80 \%)$ & $10(59 \%)$ \\
\hline \multicolumn{4}{|l|}{ Ethnicity/Race n (\%) } \\
\hline Caucasian & $10(45 \%)$ & $3(60 \%)$ & $7(41 \%)$ \\
\hline Black & $4(18 \%)$ & $1(2 \%)$ & $3(18 \%)$ \\
\hline Asian & $2(9 \%)$ & $0(0 \%)$ & $2(12 \%)$ \\
\hline Latin & $3(14 \%)$ & $0(0 \%)$ & $3(18 \%)$ \\
\hline Other & $3(14 \%)$ & $1(20 \%)$ & $2(12 \%)$ \\
\hline
\end{tabular}

a OSA defined as a mixed obstructive apnea-hypopnea index, MOAHI $\geq 1 / \mathrm{h}$

bNo OSA defined as a MOAHI $<1 / \mathrm{h}$ 
Citation: Mograss MA, Mok E, Constantin E. Evaluating Recognition Memory in Children Referred for Suspected Obstructive Sleep Apnea. J Neurol Psychol. 2015;3(2): 6.

ISSN: 2332-3469

Table 2: Polysomnographic respiratory data \& sleep variables.

\begin{tabular}{|c|c|c|c|c|}
\hline & $\begin{array}{c}\text { Total } \\
\text { Subjects } \\
(\mathrm{N}=22)\end{array}$ & $\begin{array}{c}\text { OSA } \\
(\mathrm{N}=5)\end{array}$ & $\begin{array}{c}\text { No OSA } \\
(\mathrm{N}=17)\end{array}$ & p-value \\
\hline \multicolumn{5}{|l|}{ RESPIRATORY DATA } \\
\hline Mixed Obstructive Apnea/Hypopnea Index (\#/h) & $3.6 \pm 13.4$ & $15.2 \pm 26.9$ & $0.3 \pm 0.3$ & $p=0.03$ \\
\hline Apnea Hypopnea Index (\#/h) & $4.8 \pm 13.3$ & $16.1 \pm 26.4$ & $1.5 \pm 2.9$ & $p=0.02$ \\
\hline Respiratory Arousal Index (\#/h) & $2.9 \pm 3.0$ & $2.4 \pm 10.4$ & $1.2 \pm 2.6$ & $p=0.02$ \\
\hline Desaturation Index (\#/h) & $3.1 \pm 11.7$ & $11.6 \pm 24.5$ & $0.6 \pm 0.6$ & $p=0.06$ \\
\hline Minimum SpO2 (\%) & $93 \% \pm 2.3$ & $92 \% \pm 3.1$ & $93 \% \pm 1.9$ & \\
\hline \multicolumn{5}{|l|}{ SLEEP DATA } \\
\hline TST (h) & $5.9 \pm 1.0$ & $5.3 \pm 1.7$ & $6.1 \pm 0.7$ & \\
\hline Stage $1(\%)$ & $10.3 \pm 12.6$ & $10.6 \pm 7.2$ & $10.2 \pm 14.0$ & \\
\hline Stage $2(\%)$ & $48.5 \pm 13.9$ & $51.4 \pm 9.6$ & $47.6 \pm 15.1$ & \\
\hline Slow-Wave Sleep (\%) & $25.1 \pm 7.5$ & $22.8 \pm 7.4$ & $25.8 \pm 7.6$ & \\
\hline REM (\%) & $14.8 \pm 4.1$ & $15.2 \pm 5.8$ & $14.6 \pm 3.7$ & \\
\hline Sleep Onset (minutes) & $17.6 \pm 15.1$ & $10.0 \pm 10.9$ & $19.9 \pm 15.6$ & \\
\hline Awakenings (number) & $27.2 \pm 10.9$ & $25.0 \pm 5.3$ & $27.8 \pm 12.1$ & \\
\hline Wake After Sleep Onset (minutes) & $49.0 \pm 37.6$ & $93.6 \pm 49.3$ & $36.6 \pm 21.0$ & $p=0.01$ \\
\hline
\end{tabular}

avalues represent mean \pm SD compared using independent t-test

'OSA defined as a mixed obstructive apnea-hypopnea index, MOAHI $\geq 1 / \mathrm{h}$

cNo OSA defined as a MOAHI $<1 / \mathrm{h}$

eSleep stages represent percentage of total sleep time, TST

'Sleep Efficiency - [TST/sleep period total, SPT (i.e. sleep onset to sleep offset)] x 100

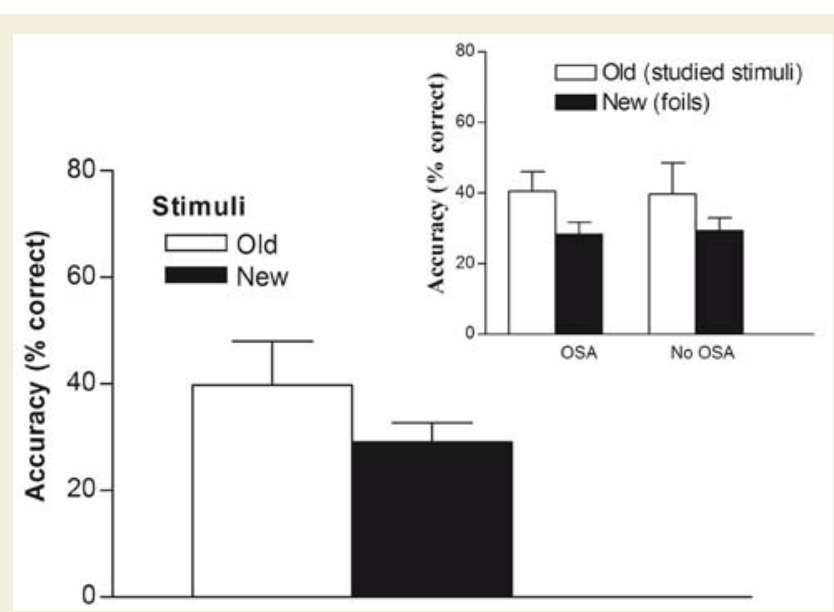

Figure 1: Mixed ANOVA to assess group differences in how accurately the children recognized the studied (Old) faces and the foils (New). There was greater accuracy at recognizing the Old vs. New faces, $p=0.001$. However, there were no differences in accuracy between the children with SDB (mixed obstructive apnea-hypopnea index, MOAHI $\geq 1 / h$ ) vs. No SDB group (MOAHI $<1 / h)$.

\section{Discussion}

This study shows that children with OSA may have retention and accuracy on a declarative memory task similar to children without OSA. Specifically we showed that in a brief, facial "recognition" memory task with a night of objectively measured sleep (PSG) between the learning/study and test session, children with mild-tosevere OSA may have similar recognition memory as those without OSA. Of particular interest and clinical relevance, we also found that longer sleep duration in the sleep laboratory was associated with increased accuracy in memory performance in children with suspected OSA, independent of OSA status.
As for our objective measure (PSG) the continuity of sleep (Table 2: TST \& number of awakenings) was not significantly different. The sleepiness scores as measured on the Epworth scale were not significantly higher in those with OSA. The ESS correlated with the parental report of the child's level of daytime sleepiness on the prescreening question of which greater than $80 \%$ of the children had symptoms of daytime sleepiness in the range of none-to-some of the time (Pearson: $r=0.68, p<0.001$ ). As for our measure of alertness prior to the experimental procedure, no difference was found between the two groups on vigilance (VAS scale for alertness) during the memory test session. What was different was that $12 \%$ of the children without OSA responses were in the "less alert" or upper third end of

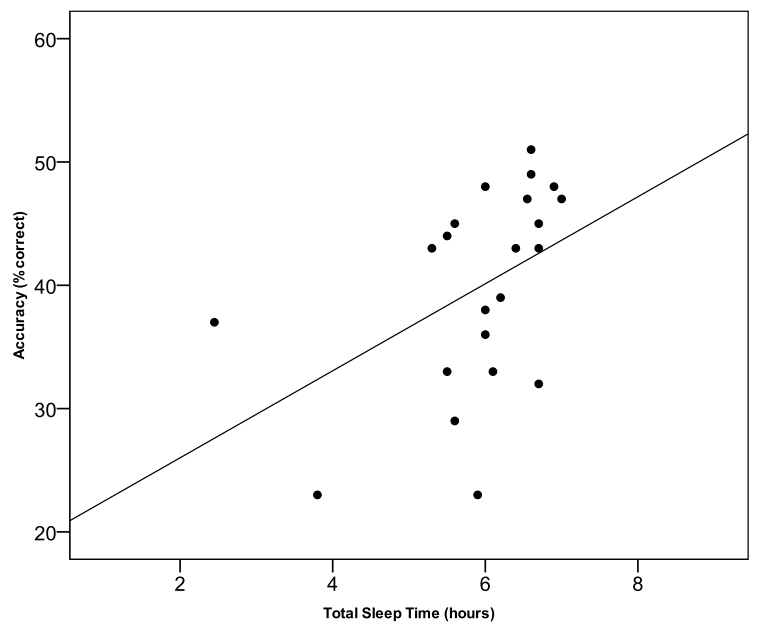

Figure 2: We observed increases in the amount of sleep associated with improvements in accuracy performance (\% correct) on the studied stimuli (Pearson : $r=0.45, p=0.04$, 2-tailed) 
the VAS scale $(>70-100 \mathrm{~mm})$ during the evening study session, and this may have influenced our results. However, this does not explain why those children with OSA, who had larger amounts of intervening wakefulness (WASO) and a lower Sleep Efficiency on the overnight sleep study, were not sleepier on the Epworth or vigilance scale (VAS) than those without. Nor does it explain why their neurobehavioral performance was not affected by the poor quality sleep. It may be that compensatory mechanisms contribute to overcoming impairments in our OSA group on our recognition memory task [28]. More research is needed to assess the amount of sleep loss that can be tolerated or that leads to negative consequences. This should aid in better understanding potential compensatory mechanisms.

Since our sleep study was performed on a weekday (vs. weekend), the amount of sleep obtained most likely reflects that usually obtained on a school night. Most children need approximately 9-10 hours of sleep for optimal alertness [29]. In those who get less sleep than what they need, their reaction time is slower which may explain our lack of group differences in RT performance. Along the same lines, in the present study, it may also explain the decreased memory performance in those children with less amount of sleep.

As mentioned in the introduction, the spectrum of disease in OSA ranges from snoring to more severe forms such as OSA. Our findings are in agreement with studies that did not find evidence of problems with memory among children with mild OSA $[9,16,17]$. Calhoun and colleagues examined the relationship between OSA \& neurocognitive function in children 6-12 y.o. These authors reported that children with mild OSA(AHI $1 / \mathrm{h}$ to $<5 / \mathrm{h}$ ) showed no significant impairment on neuropsychological tests compared to children without OSA (AHI $<1 / \mathrm{h}$ ) [30] when recalling information (words). This would indicate that in all subtypes of declarative memory (recall and recognition tasks) children with mild OSA performance is similar to those without.

In children with mild-to-moderate OSA, Friedman et al. reported impairment in neurocognitive tests involving immediate short term recall compared with healthy controls. However, there were no correlations between performance and the severity of OSA (respiratory disturbance index, desaturations or sleep fragmentation) [7]. Similarly, in the present study we found no correlations between performance (accuracy, RTs) and the severity of OSA as measured by AHI, SAO2 desaturation index, respiratory-related arousals, WASO or awakenings in a long-term "recognition" memory task.

Our results are in contrast to reports of positive associations between severe forms of OSA and deficits in neuropsychological functioning in children [31-33]. This may be due to methodology issues such as range of severity of OSA in our children, the type of task (recognition vs. recall) or in the task length (15 min vs. hours). One could argue based on our sample size that we were underpowered. However, even in studies with larger sample sizes there was little evidence of deficits in recall among children with OSA $[9,13,14,16,17,34]$.

The mechanism by which OSA is proposed to affect neurocognitive functioning includes repeated episodes of hypoxia [35] and sleep disruption resulting from multiple respiratory-related arousals [36]. Compared to those without OSA, the children in our OSA group had significant differences in the number of respiratory events, respiratory arousals and a trend in the desaturation index but no difference in the minimum SpO2 levels, sleep stages, sleep efficiency or number of awakenings. In the present study our group of children with OSA did not show all of the kinds of symptoms usually accounted for by severe forms of OSA related to sleep disturbance, and may have contributed to the lack of differences in results on our task of recognition memory.

\section{Strengths and Limitations}

As mentioned in the Introduction, in several of the studies that used PSG the sleep period occurred weeks after the task was administered instead of following the learning situation. The present study addresses this and other limitations, and is novel because we administered a brief ( 15 minute) memory task (vs. a lengthy test battery) and the test session was performed in the morning immediately after the sleep period. Furthermore, the facial recognition memory task used in our study does not require a high level of verbal fluency needed in most recall tasks.

Although sample size was limited in the current study, all children underwent a full overnight PSG (the gold standard) to confirm exposure (OSA) and to obtain other sleep and respiratory measures. In addition to using an objective measure of sleep (overnight PSG), the sleep period occurred immediately following the learning situation in all children, thus reducing measurement variability. Furthermore improvements in memory were based on child's neurobehavioral performance, therefore minimizing potential for misclassification often seen in parental reports of the child's sleep habits.

Disordered breathing during sleep is an important disease of public health significances that increases a child's susceptibility to: (1) impaired school performance, (2) risk for unintentional injury due to fatigue, and (3) risk for health-related problems associated with reduced sleep quantity such as obesity, diabetes, and adult cardiovascular disease. The contribution that this research will make to the advancement of knowledge is a better understanding of how a reduction in total sleep time may influence memory deficits in certain subtypes of declarative memory.

In conclusion, our study did not find a clear association between OSA and deficits in memory performance, when using a brief recognition memory task. We did however find a modest association between increased sleep duration and higher accuracy in memory performance. Future studies should determine whether sleep quantity in OSA is relevant in preventing a child's susceptibility to impairments in recognition and other types of memory.

\section{References}

1. Marcus CL (2001) Sleep-disordered breathing in children. Am J Resp Criti Care Med 164: 16-30.

2. Witmans $M$, Young R (2011) Update on pediatric sleep disordered breathing Pediatr Clin North Am 3: 571-89.

3. Kirk V, Kahn A, Brouillette RT (1998) Diagnostic approach to obstructive sleep apnea in children. Sleep Med Rev 2: 255-269.

4. Marcus CL (2004) Pediatric sleep medicine comes into its own. Pediatrics 113: 1393-1394.

5. Nixon GM, Kermack AS, Davis GM, Manoukian JJ, Brown KA, et al. (2004) Planning adenotonsillectomy in children with obstructive sleep apnea: the role of overnight oximetry. Pediatrics 113: e19-e25.

6. Quan SF, Goodwin JL, Babar SI, Kaemingk KL, Enright PL, et al. (2003) Sleep architecture in normal Caucasian and Hispanic children aged 6-11 years recorded during unattended home polysomnography: experience from the Tucson Children's Assessment of Sleep Apnea Study (TuCASA). Sleep 
Citation: Mograss MA, Mok E, Constantin E. Evaluating Recognition Memory in Children Referred for Suspected Obstructive Sleep Apnea. J Neurol Psychol. 2015;3(2): 6.

Med 4: 13-19.

7. Friedman BC, Hendeles-Amitai A, Kozminsky E, Leiberman A, Friger M, et al. (2003) Adenotonsillectomy improves neurocognitive function in children with obstructive sleep apnea syndrome. Sleep 26: 999-1005.

8. Kheirandish-Gozal L, Gozal D (2008) The multiple challenges of obstructive sleep apnea in children: diagnosis. Curr Opin Pediatr 20: 650-653.

9. Owens J, Spirito A, Marcotte A, McGuinn M, Berkelhammer L (2000) Neuropsychological and behavioral correlates of obstructive sleep apnea syndrome in children: A preliminary study. Sleep Breath 4: 67-78.

10. Guilleminault C, Korobkin R, Winkle R (1981) A review of 50 children with obstructive sleep apnea syndrome. Lung 159: 275-287.

11. Guilleminault C, Eldridge FL, Simmons FB, Dement WC (1976) Sleep apnea in eight children. Pediatrics 58: 23-30.

12. Kheirandish L, Gozal D (2006) Neurocognitive dysfunction in children with sleep disorders. Dev Sci 9: 388-399.

13. Archbold KH, Giordani B, Ruzicka DL, Chervin RD (2004) Cognitive executive dysfunction in children with mild sleep-disordered breathing. Biol Res Nurs 5 : 168-176.

14. Blunden S, Lushington K, Lorenzen B, Martin J, Kennedy D (2005) Neuropsychological and psychosocial function in children with a history of snoring or behavioral sleep problems. J Pediatr 146: 780-786.

15. Chervin RD, Ruzicka DL, Giordani BJ, Weatherly RA, Dillon JE, et al. (2006) Sleep-disordered breathing, behavior, and cognition in children before and after adenotonsillectomy. Pediatrics 117: e769-e778.

16. Beebe DW, Wells CT, Jeffries J, Chini B, Kalra M, et al. (2004) Neuropsychological effects of pediatric obstructive sleep apnea. J Int Neuropsychol Soc 10: 962-975.

17. O'Brien LM, Mervis CB, Holbrook CR, Bruner JL, Klaus CJ, et al. (2004) Neurobehavioral implications of habitual snoring in children. Pediatrics 114 44-49.

18. Franco RA Jr, Rosenfeld RM, Rao M (2000) First place--resident clinical science award 1999. Quality of life for children with obstructive sleep apnea. Otolaryngol Head Neck Surg 123: 9-16.

19. Johns MW (1991) A new method for measuring daytime sleepiness: the Epworth sleepiness scale. Sleep 14: 540-545.

20. Kaminska M, Jobin V, Mayer P, Amyot R, Perraton-Brillon M, et al. (2010) The Epworth Sleepiness Scale: self-administration versus administration by the physician, and validation of a French version. Can Respir J 17: e27-e34.

21. Mograss M, Godbout R, Guillem F (2006) The ERP old-new effect: A usefu indicator in studying the effects of sleep on memory retrieval processes. Sleep 29: 1491-1500.

22. Mograss MA, Guillem F, Godbout R (2008) Event-related potentials differentiates the processes involved in the effects of sleep on recognition memory. Psychophysiology 45: 420-434

23. Mograss MA, Guillem F, Stickgold R (2010) Individual differences in face recognition memory: comparison among habitual short, average, and long sleepers. Behav Brain Res 208: 576-583.

24. Debruille JB, Brodeur MB, Hess U (2011) Assessing the way people look to judge their intentions. Emotion 11: 533-543.

25. Brouillette RT, Jacob SV, Waters KA, Morielli A, Mograss M, et al. (1996) Cardiorespiratory sleep studies for children can often be performed in the home. Sleep 19(10 Suppl): S278-S280.

26. American Thoracic Society (1996) Standards and indications for cardiopulmonary sleep studies in children. American Thoracic Society. Am J Respir Crit Care Med 153: 866-878.

27. Hobson AJ (1968) A manual of standardized terminology techniques and scoring systems for sleep stages of human subjects. Rechtschaffen $A$, Kales A (Editors), Public Health Service, US Government Printing Office, Washington, DC 26: 644

28. Carskadon MA, Harvey K, Dement WC (1981) Sleep loss in young adolescents. Sleep 4: 299-312.

29. Iglowstein I, Jenni OG, Molinari L, Largo RH (2003) Sleep duration from infancy to adolescence: reference values and generational trends. Pediatrics 111: 302-307.

30. Calhoun SL, Mayes SD, Vgontzas AN, Tsaoussoglou M, Shifflett LJ, et al. (2009) No relationship between neurocognitive functioning and mild sleep disordered breathing in a community sample of children. J Clin Sleep Med 5: 228-234.

31. Galland BC, Taylor BJ, Elder DE, Herbison P (2012) Normal sleep patterns in infants and children: a systematic review of observational studies. Sleep Med Rev 16: 213-222.

32. Halbower AC, Degaonkar M, Barker PB, Earley CJ, Marcus CL, et al. (2006) Childhood obstructive sleep apnea associates with neuropsychological deficits and neuronal brain injury. PLoS Med 3: e301.

33. O'Brien LM, Gozal D (2004) Neurocognitive dysfunction and sleep in children: from human to rodent. Pediatr Clin North Am 51: 187-202.

34. O'Brien LM, Mervis CB, Holbrook CR, Bruner JL, Smith NH, et al. (2004) Neurobehavioral correlates of sleep-disordered breathing in children. J Sleep Res 13: 165-172.

35. Bedard MA, Montplaisir J, Richer F, Rouleau I, Malo J (1991) Obstructive sleep apnea syndrome: pathogenesis of neuropsychological deficits. J Clin Exp Neuropsychol 13: 950-964

36. Chugh DK, Weaver TE, Dinges DF (1996) Neurobehavioral consequences of arousals. Sleep 19(10 Suppl): S198-S201.

\section{Acknowledgements}

This study was supported by funding from the Department of Pediatrics, McGill University, the Research Institute of the McGill University Health Centre (RI-MUHC), and the Foundation of Stars/Fondation des êtoiles. MAM was supported by a Banting post doctoral fellowship from the Canadian Institutes of Health Research. EC is supported by an investigator award from the Fonds de recherche du Québec-Santé. We thank our sleep laboratory staff for the data collection as well as the parent(s) and children who participated in the study. 